Diánoia, vol. 35, no. 35, 1989

\title{
¿QUÉ MIDEN LOS JUEGOS DE LENGUAJE?
}

KUNO LORENZ

UNIVERSIDAD DE SAARBRÜCKEN

Para entender la función de los juegos de lenguaje resulta muy útil examinar de cerca una de las metáforas más importantes de las que se sirvió Wittgenstein a lo largo de su vida. Mi propósito principal será explicar cómo el término 'Bild' (figura) cambió de significado al pasar del Tractatus $(T)$ a las Philosophische Untersuchungen (PU) [Investigaciones filosoficas], mediante la determinación precisamente de aquella función de 'Bild' que se mantuvo constante. Permítaseme comenzar con el hecho bien sabido de que 'Bild' es algo al nivel de las oraciones, tanto en el $T$ como en las $P U$ : en sus Cuadernos de notas (29.9.14), Wittgenstein escribe: “en la oración se conjunta un mundo de manera experimental. (Como cuando en el juzgado en París se representa un accidente automovilístico con ayuda de muñecos, etc.)". Y en el $T$ continúa (4.031l y s.): "un nombre toma el lugar de una cosa y otro el lugar de otra y están unidos entre sí. Así el todo muestra - como una figura viva - la situación objetiva. La posibilidad de las oraciones descansa en el principio del remplazo (Vertretung) de los objetos por los signos". Así mismo leemos en las $P U(\S 23)$ : "imaginemos una figura que represente un boxeador en una pose particular. Ahora bien, esta figura puede usarse para indicarle a alguien cómo debiera posar, qué actitud debiera adoptar; o bien, qué actitud no debería de adoptar; o cómo posaba un hombre particular en tal y cual lugar; y así. Uno podría [...] llamar a esta figura un radical de oraciones". Desde luego, hay aquí un cambio ligero pero significativo que de inmediato nos esforzaremos por notar. En la cita del $T$, la figura representa una situación objetiva a la manera de las oraciones, mientras que en la cita de las $P U$, la figura puede ser usada, por ejemplo, para representar una situación objetiva, pero también con otros propósitos - como ocurre con los radicales de oraciones, que al usarse se complementan con sus modos y generan aseveraciones, preguntas, órdenes, etc. De este modo, parece (y así suelen proceder las teorías de los actos de habla) como si hubiera un acto locucionario básico, "la predicación", que se ejecuta mediante "núcleos proposicionales", y que en las verbalizaciones, es decir, en las oraciones en uso, aparece como significado locucionario junto con fuerza ilocutoria y perlocutoria. Se dice que Wittgenstein se ocupó 
en su $T$ sólo del significado locucionario, pero que dejó de lado esta noción en favor de un estudio de los actos de habla con predicación, que en este contexto podríamos llamar constataciones o aseveraciones, y que son sólo algunos entre aquéllos.

Por ello, más que ocuparnos de sólo un juego de lenguaje que dice algo acerca de los objetos como una función de sus nombres - como se dice que Wittgenstein lo hizo en el $T$-, la arena está abierta para el estudio de una variedad ilimitada de tales juegos; tal sería en efecto la opinión de Wittgenstein, aunque los teóricos de los actos de habla pretenden reducir dicha variedad a cinco tipos diferentes.

Creo que esta tesis distorsiona las ideas de Wittgenstein. Puede conjeturarse que ella se dio porque pasó más o menos desapercibido el cambio de significado de algunos términos cruciales, que está implícito en cl paso de un nivel de explicación a un nivel descriptivo, que a su vez, según las palabras del propio Wittgenstein, ocurrió entre el $T$ y las $P U$. Me refiero no solamente al término 'Bild', sino también al término 'Satz', entre los que se mantiene una relación recíproca casi inalterada aunque ella se ha trasladado a otro nivel -ustedes recordarán: mientras una figura es como una oración en el $T$, en las $P U$ funciona como un radical de oraciones. Usando una terminología peirceana, sostengo que una figura (así como una oración) funciona como una representación simbólica en el $T$, pero que - junto con los radicales de oración- se comporta como un medio de representación icónica en las $P U$. Esto implica que los juegos de lenguaje son la versión wittgensteineana de las representaciones icónicas, y que el término 'juego de lenguaje' no puede aplicarse con propiedad a la teoría de la figuración del $T$.

Para confirmar lo que sostengo, quiero csbozar antes lo que en otra parte he defendido en detalle: en el $T$, las oraciones representan una situación objetiva debido a que son representantes de una clase de abstracciones con respecto a la relación de equivalencia entre oraciones que se expresa mediante las reglas de traducción. Verter la teoría de la figuración del $T$ de este modo como una teoría de la abstracción, obviamente implica que las figuras del $T$ resulten representaciones simbólicas. Ahora las razones. El punto de partida es el bien conocido pasaje de los Cuadernos de notas (27.10.14): "la dificultad de mi teoría de la figuración lógica consistió en hallar una conexión entre los signos sobre el papel y la situación allá afuera en el mundo. Siempre dije que la verdad es una relación entre la proposición y la situación, pero nunca pude dar con ella". Con más generalidad todavía, dos meses antes (3.9.14) declaraba: "obviamente, hay oscuridad en la pregunta: ¿en qué consiste realmente la identidad lógica de signo y cosa significada? Y esta pregunta es (una vez más) un aspecto principal de todo el problema filosófico".

Ustedes saben bien que en la época en que realmente fue escrito el $T$, la identidad lógica entre signo y cosa significada se había convertido en una re- 
lación interna que se muestra a sí misma y que no puede ser dicha, mientras que la relación entre proposición y situación que en vano buscaba Wittgenstein es una relación externa que en verdad no existe. Ya aquí hemos rastreado el germen de uno de los puntos de vista más básicos y que todavía ahora no ha sido reconocido de lleno por todos los que trabajan en esta área: es un descubrimiento de Wittgenstein el que la cópula, que vincula un signo a uno o más objetos para efectuar una predicación, no es una relación ordinaria entre objetos; es más bien el medio por el que podemos enunciar que se da tal relación ordinaria.

La distinción entre 'interno' y 'externo', que se usa de manera más o menos sinónima con la distinción tradicional entre 'formal' y 'material', se convierte en una herramienta; ésta sirve para caracterizar con claridad lo que significa permanecer en el nivel objeto (para tratar objetos, propiedades, relaciones -todos ellos externos) y qué significa exhibir los medios mismos que se requieren para hacer disponibles los objetos, las propiedades, etc. Esos medios están internamente relacionados con objetos, etc.; ellos son instrumentos puramente semióticos o "símbolos", que no han de confundirse con los portadores materiales de los símbolos, por ejemplo sonidos, que reciben su función por convención, que es una relación externa.

Una serie de pasajes relevantes del $T(4.124,2.0123,2.01231,2.0141)$ nos permiten derivar: (1) conocer un objeto equivale a conncer todas sus propiedades internas; (2) conocer un objeto equivale a conocer todas las posibilidades de su ocurrencia en situaciones objetivas; (3) las formas de un objeto son posibilidades de su ocurrencia en situaciones objetivas. Estos tres enunciados implican: las propiedades internas de un objeto son sus formas, es decir, sus propiedades formales. Y estas formas de los objetos determinan completamente las estructuras de aquellas situaciones objetivas en las que ellos pueden ocurrir. Las situaciones objetivas carecen de propiedades externas, lo que explica por qué deben distinguirse de los objetos. Consideremos ahora el pasaje central en el $T(4.01)$, donde Wittgenstein caracteriza la relación interna entre el lenguaje y el mundo. Comúnmente se considera que dicho pasaje demuestra la tesis de un isomorfismo entre ambos, pero ciertamente los isomorfismos son especies de relaciones externas entre objetos. Cito:

La oración es una figura de la realidad. ${ }^{1}$ La oración es un modelo de la realidad según pensamos que ella es. A primera vista, la oración - digamos, tal como esrá impresa sobre papel - no parece ser una figura de la realidad de la que trata.

1 Como las oraciones (sentences) son signos-de-oración, es decir, tipos de objeto físico -y no ejemplares (tokens) - que, tomados junto con su función significativa han de verse como símbolos, esa expresión normalmente se traduce al inglés como 'proposiciones' (propositions) lo cual, aunque recibió la aprobación de Wittgenstein, desafortunadamente incita al lector a distraerse con la discusión superflua sobre el estatus ontológico de dichas entidades. 
Pero tampoco la partitura parece ser una figura de una pieza de música [...] Y sin embargo, esos simbolismos resultan sin duda ser figuras -en el sentido ordinario de la palabra - de lo que ellos representan [... ] El disco del gramófono, el pensamiento musical, la partitura, las ondas sonoras, todos ellos se encuentran recíprocamente en esa relación interna de figuración que existe entre el lenguaje y el mundo. Todos ellos tienen en común la estructura lógica.

Permítaseme apuntar que 'estructura lógica', un término que no aparece en otro lugar del $T$, debe leerse como 'forma lógica de la representación' (= forma de la realidad); distintas figuras equivalentes tienen diferentes formas de representación, pero son iguales en su forma lógica. El pasaje continúa refiriéndose a las reglas de traducción entre distintas figuras, que establecen su equivalencia mutua - 'similitud interna' la llama Wittgenstein, quien concluye con las siguientes palabras: "para entender la esencia de la oración, consideremos la escritura jeroglífica, que figura los hechos que describe". Ahora debemos añadir que "el que tales propiedades y relaciones internas tengan vigencia no puede, sin embargo, aseverarse mediante oraciones, sino que se muestra a sí mismo en las oraciones que representan las situaciones objetivas y tratan de los objetos en cuestión" (4.122). Por eso, la relación interna entre el lenguaje y el mundo se muestra a sí misma en las oraciones y no puede convertirse a su vez en el objeto de meta-oraciones. De hecho, tal como nos lo enseñaron las notas de cátedra de Moore (1930-1933), Wittgenstein considera que las relaciones internas son gramaticales, lo que es su término para designar lo que hoy llamaríamos 'relaciones semióticas'. Wittgenstein mismo da razones para su uso del término 'Bild' para designar la relación interna entre una oración y una situación objetiva: se refiere al hecho de que una oración se entiende solamente sobre la base de nuestro conocimiento de los objetos acerca de los cuales la oración dice algo; y aquí, 'conocimiento' es conocer las propiedades internas, es decir, aquellas por las que se "definen" los objetos. Una figura puede ser verdadera o falsa, he ahí por qué puede ser llamada un modelo de la realidad (2.1512): "es como la vara de medir que se aplica a la realidad". Aquí, también, se sugiere por sí misma la idea correspondiente que concierne a los juegos de lenguaje: en las $P U, \S 130$ y s., Wittgenstein declara que "los juegos de lenguaje se erigen más bien como objetos de comparación de los que se pretende arrojen luz sobre los hechos del lenguaje no sólo por la vía de las similitudes sino también por la de las diferencias. Pues podemos evitar la ineptitud o la vacuidad de nuestras ascveraciones sólo presentando al modelo como lo que es, como un objeto de comparación - como una vara de medir, por así decirlo". Y, de nuevo, la diferencia sutil pero decisiva: en las oraciones del caso $T$ las varas de medir son portadoras de valores de verdad, mientras en el caso $P U$ los juegos de lenguaje, siendo varas de medir, nos dicen lo que hay. Nos hemos movido de un nivel epistemológico a otro ontológico. 
Regresemos una última vez a la argumentación del $T$ : sabemos ya que las oraciones, al ser medios para decir algo acerca de objetos (es decir, para describir/representar situaciones objetivas), no son nombres de nada, sino que - como lo escribe en sus Cuadernos de notas (21.11.14) - "las realidades que corresponden al sentido de una oración son sólo sus partes componentes" y por tanto, los objetos acerca de los que se dice algo están del otro lado del lenguaje, no el de las situaciones objetivas: lo que se dice sobre ellas, que una situación objetiva es vigente, es decir, que es un hecho, tan sólo pertenece al lenguaje. De aquí proviene una distinción entre el mundo (la totalidad de lo real) y la sustancia del mundo.

Ahora bien, las oraciones tienen aspectos esenciales, es decir, propiedades internas que son comunes a todos los signos-de-oración que expresan un mismo sentido. $\mathrm{Y}$ acerca de todos los símbolos, sean nombres u oraciones, Wittgenstein apunta que (3.344): "lo que significa en el símbolo es lo que es común a todos aquellos símbolos por los que puede ser sustituido de acuerdo con las reglas de la sintaxis lógica”. Llegamos de nuevo a las reglas de traducción que eventualmente definen qué significa tener el mismo significado: "para reconocer al símbolo en el signo, hemos de considerar el uso significativo. El signo determina una forma lógica sólo unido a su aplicación lógico-sintáctica".

El lenguaje ideal postulado por Wittgenstein en el $T$ debiera considerarse como un lenguaje comprehensivo al que perteneciera todo lenguaje simbólico bien establecido. Entonces, las reglas de traducción son parte de la sintaxis lógica del lenguaje ideal y pueden usarse como una relación de equivalencia para definir situaciones objetivas mediante abstracción. Todas las clases de significado pueden efectivamente ser definidas de esta manera. Y Wittgenstein realmente tuvo éxito al eliminar toda referencia al significado, al establecer las reglas de la sintaxis lógica (3.33): "en la sintaxis lógica, el significado de un signo no debiera nunca jugar ningún papel; ella debe poder establecerse sin hacer con ello ninguna mención del significado de un signo". Por tanto, (3.334): "las reglas de la sintaxis lógica deben ser inteligibles por sí mismas, con sólo conocer cómo significa cada signo particular".

Podemos concluir que el sentido de las oraciones queda completamente explicado por el uso que hacemos de ellas, es decir, por las reglas sintácticas, siempre y cuando se sepa qué es lo que se muestra cuando usamos una oración. Y este conocimiento de qué se muestra al usar oraciones es lo que se convierte en la preocupación de Wittgenstein en las $P U$. Los juegos de lenguaje sirven como una especie de "reconstrucción racional" para mostrar (dando conocimiento activo) qué se muestra a si mismo (conocimiento pasivo) en el $T$. Cuando dice Wittgenstein, como he citado más arriba, que los juegos de lenguaje son una vara de medir para los hechos de nuestro lenguaje, no son "hechos" preexistentes (en alemán: Verbältnisse) los que estos juegos ponen 
en foco. Estos juegos más bien proveen “representaciones perspicuas" (§ 122) - término que de hecho es muy cercano a mi afirmación de que los juegos de lenguaje son representaciones icónicas-a las que Wittgenstein considera como fundamentales. De este modo se enfatiza el carácter semiótico de los juegos de lenguaje además de su aspecto obviamente pragmático.

Más que ser medios para hablar acerca de los objetos, los juegos de lenguaje muestran con qué clase de objetos estamos tratando y de qué manera. Ellos articulan situaciones al exponer su función entre las personas. Un juego de lenguaje es a la vez una perspectiva del mundo y una forma de vida. Esta idea coincide con una intuición presente ya en Platón cuando, en su Cratilo, habla de las dos características del habla humana, su función significativa y su función comunicativa. De hecho, cada verbalización muestra dos aspectos: significa y comunica, esto es, juega el papel de un término (o una palabra) y el papel de una oración; lo que es bien conocido en el caso más simple de las oraciones que consisten en una sola palabra, como en los famosos ejemplos de juegos de lenguaje de las secciones iniciales de las $P U$, donde las expresiones 'bloque', 'columna', 'losa', etc. cumplen ambas funciones.

Los juegos de lenguaje son una especie de actividad que pretende poner de manifiesto qué está sucediendo, dándonos instrumentos de comparación. Así, no sólo observamos y describimos acciones y actos-signo de acuerdo con ciertos patrones, sino también los producimos en una forma ordenada - perspicuamente, como antes habíamos notado-, para llegar a alguna clase de reconstrucción aproximada de lo que tomamos como ya disponible. Un juego de lenguaje puede considerarse como un caso paradigmático de conocimiento perceptual, en cuanto su función significativa se lleva a cabo por ser un ícono en el sentido de C. S. Peirce: hemos hallado un área de objetos internamente estructurados al inventar un prototipo. $\mathrm{Y}$ resulta obvio el que aún la distinción entre acción y acto-signo, que es un caso especial de la incómoda distinción básica entre el mundo y el lenguaje, debe ser relativizada en consideración de una versión puramente funcional de lo que significa ser un objeto y lo que significa ser el signo de un objeto.

Debemos intentar, por tanto, el desarrollo de un acercamiento unificado tanto a los objetos como a sus signos, muy en consonancia con la metodología pragmática de Peirce, de que una teoría de la designación y una teoría de los designata deben ser tareas combinadas. Mi propuesta es girar hacia un modelo del diálogo donde se parta de una situación de adquisición de una competencia de acción, a la vez que de una competencia de acto-signo. Como un paso preliminar, les pido considerar el lenguaje verbal y otros sistemas simbólicos en uso, primordialmente como tipos de acciones tales como comer o dormir, con la consecuencia de que en este respecto ellos mismos son partes de los objetos a los que se refieren; y complementariamente, tengan ustedes a los objetos no lingüísticos y especialmente a los no simbólicos, como partes 
de una red de acciones interrelacionadas e interdependientes; así se toma en cuenta el que ellos participen del valor significativo de las actividades en las que están inmersos.

Por tanto, lo que hacemos inicialmente es naturalizar al lenguaje incluyendo otros sistemas simbólicos, y simbolizar el mundo al poner atención a aquel aspecto de las acciones que se pone en foco cuando reconocemos las acciones más que simplemente llevarlas a cabo. El puente sobre el aparente hiato entre mundo y lenguaje puede construirse si hacemos desaparecer el hiato. Las acciones son tanto ejecutadas - lo que es su aspecto natural o empírico-como comprendidas, en lo que consiste su aspecto simbólico o racional. Desde el primer punto de vista, producimos ejemplares (tokens) de un tipo, y desde el segundo, "identificamos" diferentes ejemplares como si pertenecieran al mismo tipo.

Ahora procederemos de una manera más sistemática, y armaremos efectivamente nuestro modelo dialógico, para conseguir una apreciación más firme de los dos aspectos fundamentales de las acciones, su aspecto realizativo y su aspecto de reconocimiento, a los que ahora llamaremos respectivamente aspectos pragmático y semiótico. No es en absoluto una idea nueva, debiera añadir, la de que a cada acción puede aplicarse la distinción entre el mero actuar y actuar como una especie de lenguaje; aunque esta división de aspectos no se haya aprovechado aún en una reconstrucción genética de la competencia lingüística, o al menos de algunas fases iniciales de tal competencia, desde los procesos de adquirir esquemas de acción (= tipos de acción).

El modelo dialógico consiste en una situación elemental de dos personas ocupadas en iniciar y seguir un proceso de adquirir la competencia para efectuar cierta acción. La situación elemental de dos personas no es más que una versión elemental y generalizada de un juego de lenguaje wittgensteineano, especialmente con respecto a su papel como vara de medición ( $P U$ § 131) para complejos de acciones ya presentes, incluyendo acciones linguiísticas. El arranque a partir de procesos de adquisición de acciones más que de conjuntos de sucesos de acción (que son alguna clase de entidades externas singulares), o de conjuntos de competencias pragmáticas (que vienen siendo una especie de entidades universales internas), proviene de la necesidad de evitar las perennes trampas del dualismo cartesiano con su incómoda consecuencia de tener que servirse ya de un enfoque conductista, ya de una perspectiva mentalista, para abordar nuestras habilidades, en particular las lingüísticas. El propósito de las situaciones de diálogo elementales junto con la construcción de una diversificación gradualmente creciente de situaciones de diálogo más y más complejas, es conservar una versión unificada de cómo logramos a la vez participar en una situación común y el uso de un sector comunitario del lenguaje. Esto se consigue por la observación cuidadosa de las dos perspectivas ejercitadas por los dos agentes en tal situación de adquisición de competencias. En un mo- 
mento dado, uno de ellos está ejecutando mientras el otro reconoce la acción, y en el curso de los procesos de adquisición, los puntos de vista se intercambian permanentemente. Como ejercicio de cautela, me inclino a añadir que los términos 'ejecutar' y 'reconocer' sólo pueden aplicarse post hoc, es decir, después de que la secuencia abierta de roles intercambiantes se ha llevado a cabo y ambos agentes han adquirido la competencia en cuestión.

Los primeros objetos que quedan disponibles con esta clase de competencia son acciones todavía sin ninguna diferenciación ulterior, ni con respecto a acto y agente ni tampoco respecto de acto y objeto del acto, ni de algún otro tipo. Pero todos ellos son y al mismo tiempo se sabe que son comunes en la situación respectiva; todavía mejor, cada uno de ellos es una situación común y ambas partes lo saben. Una vez más podrá pensarse en la famosa situación del conejo en las $P U$ de Wittgenstein (II:XI), donde en el curso de la discusión de 'ver algo' y 'ver como', pone el ejemplo de la exclamación: 'iun concjo!', con la que un paisaje donde hay un conejo corriendo se transforma en una situación-de-concjo. A este "destellar de un aspecto" lo describe como "una experiencia a medias visual, a medias pensamiento". Claro está que aquí tenemos una situación dialógica más avanzada, en cuanto el hablante que exclama 'iun conejo!' incorpora ambas partes de nuestra situación elemental, junto con algunos desarrollos significativos ulteriores: en tanto parte ejecutora, el hablante lleva a cabo una acción perceptual, mientras que como parte que reconoce profiere 'iun conejo!', y la adquisición de "la competencia-de-conejo" resulta ser cuestión de un paso único y súbito. Por otro lado, en el caso del reporte 'un conejo', confiamos en una situación perceptualmente ya definida, por ejemplo, se recuerdan conejos de ocasiones anteriores.

Para mantener separadas las distintas fases del desarrollo de nuestro modelo dialógico, es mejor que al mundo común elemental y más bien primitivo lo consideremos como consistente en pre-acciones. A ellas no pertenecen ni las cosas, ni las personas, ni los conejos en el sentido usual. Mirando atrás desde una fase posterior podrá decirse que cosas, personas, animales, etc. son todavía partes indistinguibles de las pre-acciones. Y es importante estar conscientes del hecho de que las pre-acciones no tienen el estatus de datos ni tampoco el de construcciones racionales, como nos daría a escoger la disyuntiva tradicional en términos del debate entre empirismo y racionalismo acerca del nivel primordial de la realidad, alternativa a la que también Wittgenstein quiso escapar. Las pre-acciones son llevadas a cabo por el agente actuante y son reconocidas por la otra parte, y eso es todo.

Dándonos cuenta ahora de que el punto de vista de la ejecución es pragmático, mientras que la perspectiva del reconocimiento es semiótica, podemos decir que nuestro modelo de la interacción dialógica elemental nos permite distinguir un aspecto pragmático y otro semiótico en cada pre-acción. Obtenemos una versión concreta del carácter sígnico de una pre-acción al mirar 
a la parte no-ejecutora en un instante particular de la situación de diálogo, como alguien que entiende la ejecución de la otra parte, por ejemplo, como una invitación a hacer "lo mismo".

Daremos ahora un paso más, al hacer del punto de vista del reconocimiento en una pre-acción, una pre-acción explícita por sí. Lo que sucederá: por ejemplo, en lugar de simplemente reconocer que Se Fuma, cjecutamos una preacción separada, digamos Ver Fumar, que vale como una percepción respecto de la pre-acción anterior. Reconocer una pre-acción es llevar a cabo una percepción de la pre-acción. Por lo tanto, en la situación dialógica elemental la persona que efectúa el Fumar está reconociendo al mismo tiempo la percepción de su contraparte. Es decir, en términos más coloquiales, sabe que quien percibe sabe él mismo lo que es Fumar gracias a que ejecuta una pre-acción perceptual con respecto a Fumar; o bien, sabe que su contraparte está sabiendo cómo Fumar.

El próximo paso consiste en identificar el aspecto de reconocimiento de una pre-acción con el aspecto realizativo de una percepción de la pre-acción, lo que puede ser llamado 'objetivación' y conduce a la fase de constitución de objetos independientes de situaciones dialógicas particulares. Podemos decir que por abstracción pragmática damos la definición de un pre-objeto - no me sirvo del término 'objeto' porque no habrá aún división alguna en unidades individuales (los individuos). Este pre-objeto es lo invariante en el conjunto abierto de percepciones relevantes, independientemente del proceso dialógico particular de adquisición de la pre-acción original.

Reconocer un pre-objeto no es sino llevar a cabo una percepción correspondiente; los pre-objetos mismos no pueden seguir siendo realizados, sino que son los mismos para todos los participantes, aunque con diferencias en su acceso perceptual. Aún las acciones como pre-objetos no llegan a ejecutarse. Lo que se ejecuta, por ejemplo, en el caso de Fumar como pre-objcto, es fumar como Mostrar-que se fuma; es decir, se presenta (en alemán, vorfirren) el fumar. Sólo al presentar acciones ellas cuentan, del mismo modo que las cosas, las personas, los sucesos, etc., como pre-objetos y no como meras preacciones -aunque todavía no hayamos desarrollado criterios para clasificar los pre-objetos en clases.

Decimos que los pre-objetos están articulados por sus percepciones. Pero es un hecho antropológico contingente el que ciertas pre-acciones perceptuales dentro de la variedad de articulaciones de un pre-objeto (es decir, las pre-acciones verbales), obtengan un estatus canónico con respecto a ser signos del pre-objeto: como una especie de síntoma, ellas representan al pre-objeto "pars pro toto". Para el siguiente paso, consistente en convertir las pre-acciones verbales en pre-objetos por su propio derecho sin privarlas por ello de su función perceptual (es decir, semiótica), retornaremos a situaciones dialógicas de segundo orden. Tales situaciones dialógicas sirven como reconstrucciones genéticas de lo que en la tradición de la lógica filosófica ha sido llamado (si- 
tuaciones de) 'predicación'. A los pre-objetos verbales o mejor, a sus resultados hablados o escritos en tanto puestos a nuestra disposición mediante la abstracción pragmática con ayuda de tales situaciones dialógicas de segundo orden, los llamo articuladores. Sabemos ya que ellos no pueden ser ejecutados en sentido estricto, sino que sólo pueden ser presentados. Emitir un articulador, o ejecutar una articulación o una predicación, digamos 'fumar' —que es la "percepción verbal" del pre-objeto Fumar- es al mismo tiempo un caso de su propio reconocimiento. Un articulador es, en tanto pre-objeto, un cabal signo simbólico, no ya uno meramente sintomático. Podemos distinguir esas dos funciones semióticas de un articulador diciendo que en su función sintomática el articulador es constitutivo de su objeto, mientras que en su función simbólica describe su objeto. Obviamente, también las figuras pueden emplearse como articuladores.

Sólo ahora hemos alcanzado el punto en el que los articuladores, siendo a la vez signos y acciones, esto es, acciones-signo o, aún mejor, acciones semióticas, pueden considerarse sujetas a los dos aspectos a los que aludí como las funciones básicas del lenguaje: significación y comunicación. Para evitar confusiones resulta aconsejable usar el término 'articulación' sólo con respecto a su función significativa, y cambiar al término 'predicación' cuando interesa la función comunicativa de la articulación. Puesto que en general ocurre que con respecto a su carácter pragmático un acto-signo es comunicativo, y que respecto de su carácter semiótico, una acción es significativa, puede decirse que al ejecutarse una percepción, se efectúa una articulación en su aspecto significativo. De aquí que el "significado" de un articulador se divida entre las distintas perspectivas de las personas que lo usan, y sólo así puede asegurarse la disponibilidad de significados comunes. Del mismo modo, una articulación en su aspecto comunicativo, es decir, una predicación como acción, se divide entre las dos diferentes maneras en que es dado - lo que nuevamente resulta de una objetivación, aquí de segundo orden, mediante la conversión del reconocimiento de una predicación en una acción independiente, a la que llamamos un modo de la predicación. El término más común en nuestros días para designar tal modo es, desde luego, 'acto de habla'. Las predicaciones ocurren siempre bajo un modo, a saber, el acto de habla entre el hablante y quien lo escucha: las situaciones dialógicas de segundo orden aparecen como los equivalentes sistemáticos para los juegos de lenguaje de Wittgenstein en sentido estricto.

Y lo que espero que haya quedado claro es que el uso del lenguaje se da siempre en dos niveles: como una percepción, esto es como una manera de presentar un objeto - la exclamación de iconcjo!, que es un caso de competencia-de-objeto- y como una concepción, es decir, como un modo de volver a referirse a otros términos ya anteriormente usados perceptualmente -el reporte del conejo, lo que es un caso de meta-competencia. 
En el $T$, Wittgenstein se ocupa de la habilidad para usar oraciones y, en general, figuras, como un caso de meta-competencia con respecto a actossigno, mientras que en las $P U$ presenta los juegos de lenguaje y el uso de figuras como un caso de competencia-de-objeto con respecto a actos-signo. En este último caso, ellos sirven como actos-signo ejemplificadores, para usar una palabra de Goodman. Si ustedes desprecian como una pieza de retórica la equivocidad que en esta forma se conecta con el término 'Bild', podrán decir, y quiero terminar mi charla de esta manera: en el $T$ las figuras son símbolos, mientras que en las $P U$ los juegos de lenguaje son figuras en uso.

Traducción de José de Teresa 